\title{
Lignin Aromatics to PHA Polymers: Nitrogen and Oxygen Are the Key Factors for Pseudomonas
}

\author{
Juan E. Ramírez-Morales, Phillip Czichowski, Volkan Besirlioglu, Lars Regestein, Korneel Rabaey, \\ Lars M. Blank, and Miriam A. Rosenbaum*
}

Cite This: ACS Sustainable Chem. Eng. 2021, 9, 10579-10590

Read Online

\section{ACCESS | Llll Metrics \& More | 回 Article Recommendations | (s) Supporting Information}

ABSTRACT: Many wild type Pseudomonas strains have the potential to contribute to the valorization of lignin in future biorefineries. Through a robust aromatic catabolism, i.e., biofunneling capacity, they can ease the inherent aromatic heterogeneity found in lignin hydrolysates and accumulate naturally marketable biopolymers like $m c l$-polyhydroxyalkanoate $(m c l$-PHA) under nitrogen limitation. Besides a comparative strain evaluation, we present fundamental research on the funneling of aromatic mixtures under specific bioprocess conditions to improve biocatalytic lignin valorization. For the most robust and best performing strain, $P$. putida KT2440, we improve the mcl-PHA accumulation from a defined aromatic mixture of $p$-coumarate, ferulate, and benzoate under technically relevant conditions by up to $40 \%$ by tailoring the nitrogen and oxygen supply. The highest $m c l$-PHA concentration $(582 \pm 41 \mathrm{mg}$ $\mathrm{L}^{-1}$ ) was obtained for a $\mathrm{C} / \mathrm{N}$ ratio of 60 for oxygen-unlimited conditions (oxygen transfer rate $>20 \mathrm{mmol} \mathrm{L}^{-1} \mathrm{~h}^{-1}$ ). In contrast, aromatic intermediates accumulated under oxygen-limited conditions at oxygen transfer rates below $10 \mathrm{mmol} \mathrm{L}^{-1} \mathrm{~h}^{-1}$. The experimental conditions were scalable into a $1 \mathrm{~L}$ stirred tank bioreactor. This study contributes to deepening our understanding of the biocatalytic capability of promising Pseudomonas strains toward downstream microbial conversions of lignin aromatics for future biorefinery applications.

KEYWORDS: Pseudomonas putida KT2440, Lignin valorization, Biological funnelling, Biorefining, Bioprocess development, Aromatic mixture

\section{INTRODUCTION}

Lignin, the most abundant aromatic polymer on the planet, promises to positively impact the transition to a sustainable biobased economy. Lignin represents a treasure chest from a chemical point of view, which can be exploited with advancing progress on biomass fractionation and depolymerization approaches. ${ }^{1}$ While chemical processes aim to prevent structural lignin degradation, preserve reactive ether bond structures enhancing functionality, and increase aromatic monomer yields by using catalytic methods, biochemical efforts based on ligninolytic enzymes found in fungi and bacteria also promise to minimize energy demand and hazardous waste generation. ${ }^{2,3}$ However, both chemical and biological approaches generate complex lignin-derived aromatic mixtures and only few of the obtained depolymerized products can be directly considered as marketable chemicals. ${ }^{4}$ To overcome these limitations, the concepts of chemocatalytic and biological funneling have been introduced for upgrading the value chain from lignin. ${ }^{1,5,6}$

The concept of biocatalytic funnelling is based on the ability of some bacterial species to converge a plethora of ligninderived aromatics into a few central intermediates like catechol and protocatechuate, which are eventually cleaved and metabolized via the $\beta$-ketoadipate pathway to access the central carbon metabolism. ${ }^{5}$ Among these bacteria, Pseudomonas strains gained special attention because of their metabolic robustness, genetic accessibility, fast growth, and high productivities. $^{5-8}$ Especially, Pseudomonas putida stands out for its ability to adapt and tolerate stressful environmental conditions $^{9}$ and the versatility of native or engineered value products from lignin-derived aromatics. $7,8,10-12$

A straightforward native product of $P$. putida are mediumchain-length polyhydroxyalkanoates ( $m c$ l-PHAs), which are an alternative to fossil-based plastic and can be used as a biodegradable polymer in medical and material applications. ${ }^{13}$ This storage molecule is only synthesized when an excess of carbon relative to other essential growth elements like nitrogen

Received: April 20, 2021

Revised: June 22, 2021

Published: July 23, 2021 
is given. ${ }^{14,15}$ Several P. putida strains have been recognized as efficient cell factories for mcl-PHA production, which have been fundamentally studied and optimized using conventional carbon sources. ${ }^{10,16}$ Additionally, important proof-of-concepts and cultivation studies for upgrading single lignin aromatics and/or lignin-derived streams into $m c l$-PHA in $P$. putida greatly improved production parameters. $5,11,17,18$ However, obtaining reproducible liquors of lignin-derived aromatics as substrates for biological funnelling studies in sufficient amounts is still difficult. Furthermore, limited methods for analysis of the heterogeneous aromatic fractions and complex catabolic repression phenomena make a detailed physiological study on mcl-PHA production from liquors obtained after lignin depolimerization very challenging. Therefore, much of the physiological research thus far focused on the evaluation of single lignin-derived aromatic compounds like benzoate, ${ }^{15,19}$ vanillate, $^{14}$ ferulate, $^{20}$ or cofeeding some of them with glycerol. $^{21}$ Thus, detailed studies toward a physiological understanding of the uptake and conversion dynamics of defined aromatic mixtures into mcl-PHA are necessary. In particular, knowledge on how the native funnelling capacity of $P$. putida is affected under nutrient limited conditions (i.e., when triggering $m c l-P H A$ accumulation) is required, as hierarchical aromatics consumption is likely to heavily affect productivities. ${ }^{22}$ Finally, a more rigorous comprehension of the impact of oxygen transfer rates on mcl-PHA production is a relevant gap to be covered, especially to encourage proper reporting of oxygen transfer conditions, which has been identified as a problem in the PHA field. ${ }^{23}$

We here present a systematic experimental approach to select, compare, and evaluate the best hosts to accumulate $\mathrm{mcl}$ PHA from different lignin-derived aromatic monomers as single and mixed carbon sources (Figure S1 of the Supporting Information, SI). This study starts with a 3-step sequential screening to evaluate the growth performance of seven wild type Pseudomonas strains on 15 lignin model compounds at two concentrations ( 5 and $20 \mathrm{mM}$ ). Additionally, we explore different scenarios of nitrogen but also oxygen limitation to expose the changes in funneling capacity of selected strains when accumulating $m c l$-PHA from a mixture composed of representative lignin-derived aromatics. Finally, online measurements of the microbial respiratory capacity via the oxygen transfer rate (OTR) and the dynamics of aromatic funneling into $m c l$-PHA facilitated a detailed bioprocess characterization. This study contributes to deepening our comprehension of the biocatalytic capability of promising Pseudomonas strains toward valorization of renewable lignin aromatics into biodegradable polymers like $m c l$-PHA.

\section{MATERIALS AND METHODS}

Bacterial Strains, Media Preparation, and General Culture Conditions. All wild type strains used in this study are listed in Table S1. Precultures from $-80{ }^{\circ} \mathrm{C}$ glycerol stocks were first prepared in LB medium followed by mineral salt medium (MSM) in shake flasks for $18 \mathrm{~h}$ at $200 \mathrm{rpm}$ and $30^{\circ} \mathrm{C}$ for all strains except for P. aeruginosa PA14 and $P$. aeruginosa PAO1. Both were grown at $37^{\circ} \mathrm{C}$. Prior to inoculation, the cells were harvested by centrifugation $(12000 \mathrm{~g}, 5$ $\mathrm{min}$ ), washed, and resuspended in a phosphate-buffered saline (PBS) solution. All experiments were performed using a defined MSM according to Hartmans et al. ${ }^{24}$ containing $3.88 \mathrm{~g} \mathrm{~L}^{-1} \mathrm{~K}_{2} \mathrm{HPO}_{4}, 1.63 \mathrm{~g}$ $\mathrm{L}^{-1} \mathrm{NaH}_{2} \mathrm{PO}_{4}, 0.1 \mathrm{~g} \mathrm{~L}^{-1} \mathrm{MgCl}_{2} \cdot 6 \mathrm{H}_{2} \mathrm{O}, 10 \mathrm{mg} \mathrm{L}^{-1}$ EDTA, $2 \mathrm{mg} \mathrm{L}{ }^{-1}$ $\mathrm{ZnSO}_{4} \cdot 7 \mathrm{H}_{2} \mathrm{O}, 1 \mathrm{mg} \mathrm{L} \mathrm{CaCl}_{2} \cdot 2 \mathrm{H}_{2} \mathrm{O}, 5 \mathrm{mg} \mathrm{L}{ }^{-1} \mathrm{FeSO}_{4} \cdot 7 \mathrm{H}_{2} \mathrm{O}, 0.2$ $\mathrm{mg} \mathrm{L}^{-1} \mathrm{Na}_{2} \mathrm{MoO}_{4} \cdot 2 \mathrm{H}_{2} \mathrm{O}, 0.2 \mathrm{mg} \mathrm{L}{ }^{-1} \mathrm{CuSO}_{4} \cdot 5 \mathrm{H}_{2} \mathrm{O}, 0.4 \mathrm{mg} \mathrm{L}^{-1}$ $\mathrm{CoCl}_{2} \cdot 6 \mathrm{H}_{2} \mathrm{O}$, and $1 \mathrm{mg} \mathrm{L} \mathrm{L}^{-1} \mathrm{MnCl}_{2} \cdot 2 \mathrm{H}_{2} \mathrm{O}$. The media was supplemented with $20 \mathrm{mM}$ glucose or the corresponding aromatic compound as carbon source. The source of nitrogen, $\left(\mathrm{NH}_{4}\right)_{2} \mathrm{SO}_{4}$, was added according to the carbon to nitrogen molar ratios $(\mathrm{C} / \mathrm{N}$ of 4,8 , 40,60 , and 80 ).

The lignin-derived monomers were purchased from Carl Roth (Karlsruhe, Germany) or Sigma-Aldrich (St. Louis, MO, U.S.A.). Sterile filtered stocks were prepared at $150 \mathrm{mM}$ in water by increasing the $\mathrm{pH}$ with $\mathrm{NaOH}$ and then neutralizing to $\mathrm{pH} 7.1$ with $\mathrm{HCl}$ and stored at $4{ }^{\circ} \mathrm{C}$.

Strain Screening Assays. Simultaneous online measurements of biomass formation were carried out via scattered light at $620 \mathrm{~nm}$ with the microbioreactor system BioLector (Beckman Coulter, Inc., U.S.A.) in 96- and 48-well plates. Each step started with a standardized inoculation procedure as described above at $\mathrm{OD}_{600}$ of 0.1 from a prewashed cell suspension in PBS solution. The same composition of MSM was kept, except for the amount of nitrogen. A general calibration of the BioLector device was carried out using glucose for every strain and a suitable detector sensitivity (gain) of 50 was chosen for all other experiments. Initial blank scattered light values were obtained to subtract the contribution of each media composition. A detailed technical description of the three screening steps is provided in the SI sec. S1.2.

Strain Comparison in Conventional Shake Flask. The best two strains selected in the screening stage were cultivated in duplicate at $30^{\circ} \mathrm{C}$ in $500 \mathrm{~mL}$ shaking flasks (diameter of $105 \mathrm{~mm}$ ) with a filling volume of $50 \mathrm{~mL}$. Experiments started at $\mathrm{OD}_{600}$ of 0.1 and were shaken at $200 \mathrm{rpm}$ for $124 \mathrm{~h}$ using a Multitron incubator shaker (Infors HT, Birsfelden, Switzerland) with an orbital throw of $2.5 \mathrm{~cm}$. The substrate consisted of the defined aromatic mixture of $p$ coumarate, ferulate, and benzoate, each at $10 \mathrm{mM}$. The amount of $\left(\mathrm{NH}_{4}\right)_{2} \mathrm{SO}_{4}$ was adjusted to reach $\mathrm{C} / \mathrm{N}$ ratios of 8 and 80 . The corresponding osmolarity of the mixed substrates was determined using a Loeser Freezing Point Osmometer. Measurements of 0.16, 0.17 , and $0.18 \mathrm{Osmol} \mathrm{kg}^{-1}$ were found for the substrate mixtures at $\mathrm{C} / \mathrm{N} 80,60$, and 40, respectively. A detailed explanation of the $\mathrm{OTR}_{\max }$ calculation in conventional shake flask can be found in the SI sec. S2.4.

All flow cytometry analyses were performed using a BD Influx flow cytometer (BD Biosciences). The mcl-PHA staining protocol was adapted from Karmann et al. ${ }^{25}$ Bacterial cells were diluted to approximately $10^{4}$ cells $\mu \mathrm{L}^{-1}$ in $50 \% \mathrm{v} \mathrm{v}^{-1}$ PBS buffer containing 5 mM EDTA. $500 \mu \mathrm{L}$ of sample was stained for 5 min with $5 \mu \mathrm{L}$ of BODIPY 493-503 stock at $20 \mu \mathrm{g} \mathrm{mL}^{-1}$. Samples were analyzed recording the forward scatter (FSC) and fluorescence intensity $\left(\lambda_{\text {ex }}\right.$ $\left.488 \mathrm{~nm}-\lambda_{\mathrm{em}} 530 \mathrm{~nm}\right)$. Gates (P1 and P2) were set from previously prepared samples containing $m c l-\mathrm{PHA}$ accumulating populations. Detailed information can be found in Figure S2.

Online Measurements of the Oxygen Transfer Rate in Shake Flask Cultivations. The methodology for obtaining the online respiratory activity and the dynamics of aromatic funnelling into $m c l$-PHA was adapted from Heyman et al. ${ }^{26}$ The commercial shaker device TOM (Adolf Kühner AG, Birsfelden, Switzerland) was used for online monitoring of the OTR in duplicates. The general procedure was combined with parallel cultivations under identical conditions to take offline samples in a Multitron orbitar shaker (Infors HT, Bottmingen, Switzerland). Both devices were set with an orbital throw of $5 \mathrm{~cm}, 100 \mathrm{rpm}$, and $30{ }^{\circ} \mathrm{C}$. The defined aromatic mixture $\mathrm{CFB}$ as substrate was combined with different amounts of $\left(\mathrm{NH}_{4}\right)_{2} \mathrm{SO}_{4}$ to reach $\mathrm{C} / \mathrm{N}$ ratios of 40,60 , and 80 in the MSM according to Hartmans et al. ${ }^{24}$ Cultivations were performed in unbaffled $250 \mathrm{~mL}$ shake flasks at an $\mathrm{OD}_{600}$ of 0.1 . Different OTRs were set by changing the filling volumes $(10,20,30$, and $50 \mathrm{~mL})$ with each master mix. Thus, a combination of 12 different conditions were evaluated. Compressed air was supplied to the TOM device at concentrations of $\mathrm{CO}_{2}$ and $\mathrm{O}_{2}$ of $0.04 \% \mathrm{v} \mathrm{v}^{-1}$ and $20.95 \% \mathrm{v} \mathrm{v}^{-1}$, respectively. The measured concentrations were corrected by an evaporation factor.

Cultivations in $1 \mathrm{~L}$ Stirred Tank Bioreactor. Two parallel cultivations were conducted in identical $1 \mathrm{~L}$ stirred-tank reactors Multifors 2 (Infors HT, Bottmingen, Switzerland) with a working volume of $0.7 \mathrm{~L}$. The temperature was controlled at $30{ }^{\circ} \mathrm{C}$. The 


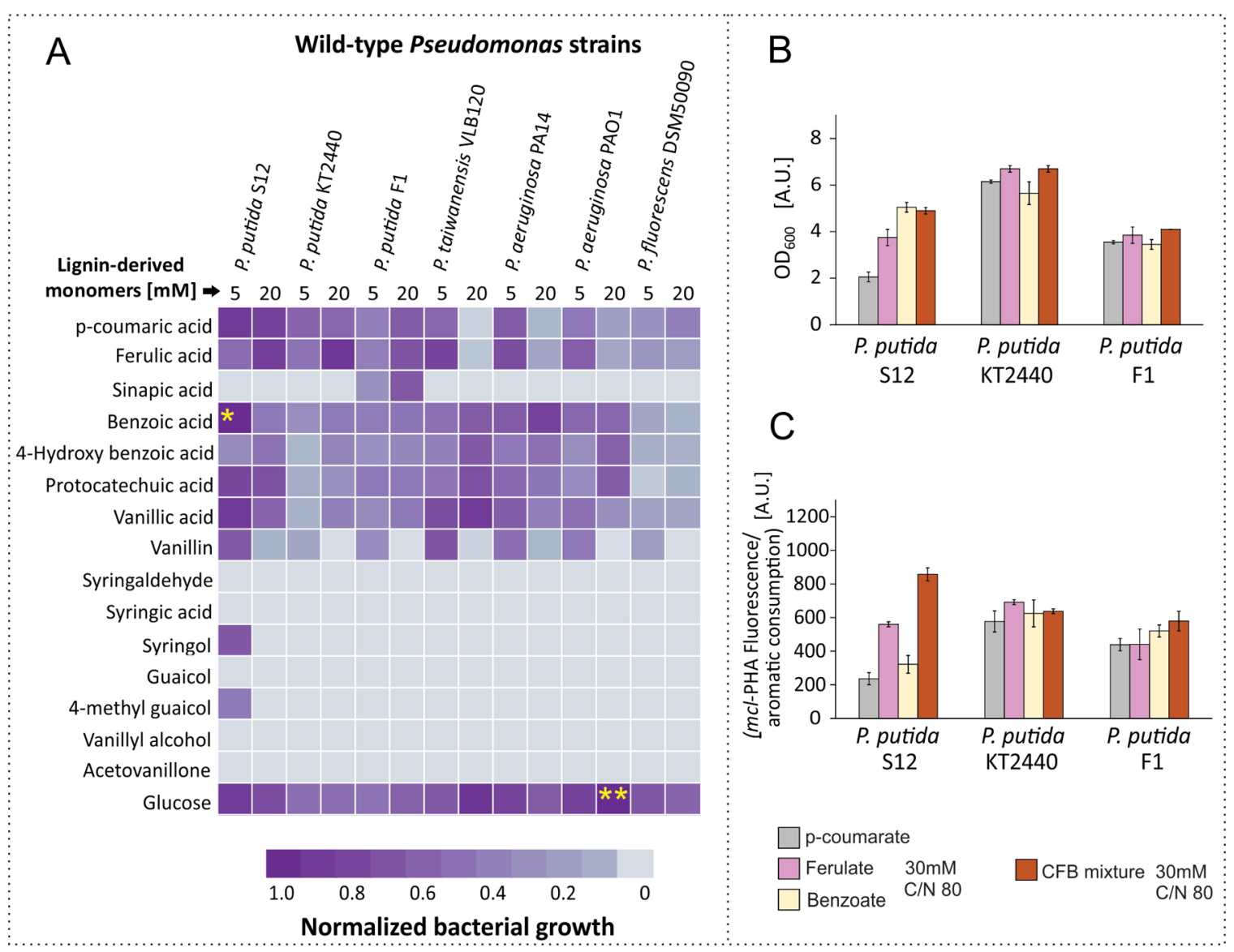

Figure 1. Screening step 1 (A) and 3 (B), (C). The first is represented as a heat map of the growth performance of 7 wild type Pseudomonas strains on 15 lignin-derived monomers at two concentrations $(5$ and $20 \mathrm{mM})(\mathrm{A})$. Glucose was used as reference carbon source and served as positive control. The strains were cultivated in microbioreactors (BioLector) using 96-well plates under nitrogen excess $(\mathrm{C} / \mathrm{N}=4)$ in Hartman's mineral salt medium for $39 \mathrm{~h}$. Normalized bacterial growth data $(\leq 1.0)$ was calculated for every experimental condition (eq S1 SI sec S2.1) in respect to the maximum scatter intensity value indicated as yellow asterisks for both aromatic concentrations $(*$ for $5 \mathrm{mM}$ : maximum scatter intensity $=183$ and **for $20 \mathrm{mM}$ : maximum scatter intensity $=578$ ). Experiments were performed in triplicates, full data are given in Data Set S1. The third screening step evaluates the valorization of an aromatic mixture with the three most suitable strains from step 2 , using a stronger nitrogen limitation $(\mathrm{C} / \mathrm{N}=$ 80) and aromatic compounds as sole carbon sources or mixed (CFB), both at $30 \mathrm{mM}$ in Hartman's mineral salt medium. The final optical density $\left(\mathrm{OD}_{600}\right)$ at $48 \mathrm{~h}(\mathrm{~B})$ and normalized $m c l$-PHA fluorescence intensity respect to the aromatic consumption (C) were measured using 48-well flower plates.

preculture and mixed substrate preparation followed the same procedure as previously described. Before inoculation, $100 \mu \mathrm{L}$ of Antifoam 204 (Sigma-Aldrich, St. Louis MO, U.S.A.) was added manually to each reactor. The initial stirring rate, $\mathrm{pH}$ and $\mathrm{OD}_{600}$ were $300 \mathrm{rpm}, 0.1$ and 7.1, respectively. To achieve the desired respiratory activity found in shake flasks, the reactors were aerated constantly with $0.175 \mathrm{~L} \mathrm{~min}^{-1}(0.25 \mathrm{vvm})$ pressurized air through mass-flow regulators. Online $\mathrm{O}_{2}$ and $\mathrm{CO}_{2}$ concentrations were measured using parallel BlueInOne Cell gas analyzers (BlueSens $\mathrm{GmbH}$, Herten, Germany). Initially, the dissolved oxygen tension (DOT) was set to a minimum level of $50 \%$ in both reactors and was controlled automatically via stirrer speed controller. The online measurements of the gas outlet composition were taken and the OTR was calculated manually using eq S6 (SI sec. S2.5). Once the desired values were achieved for every reactor, the control loop was opened and the stirrer speed was set at the corresponding constant value for the rest of the cultivation, completing $96 \mathrm{~h}$. The carbon dioxide transfer rate (CTR) and the respiratory quotient (RQ) were calculated according to eq $\mathrm{S} 7$ and eq S8 (SI sec. S2.5).

Bacterial Growth and mcl-PHA Fluorescence Measurements. Cell growth was monitored as optical density at $600 \mathrm{~nm}$ (OD600) with an Ultrospec 10 spectrophotometer (GE Healthcare, Chicago, IL, U.S.A.) and online measurements of back scattered light, carried out during the screening assays. In cases where enough sample was available, cell dry weight (CDW) was also determined.

Mcl-PHA fluorescence measurements were performed using BODIPY 493/503 (Thermo Fisher, U.S.A.). ${ }^{27}$ The staining procedure was modified as published by Karmann et al. ${ }^{25}$ The cells were harvested by centrifugation $(12000 \mathrm{~g}, 5 \mathrm{~min})$, washed and resuspended in $300 \mu \mathrm{L}$ of $50 \% \mathrm{v} / \mathrm{v}$ PBS buffer containing $5 \mathrm{mM}$ EDTA to a final OD of 1.5 . A stock solution of BODIPY 493/503 dissolved in DMSO was added to reach a final concentration of 0.67 $\mu \mathrm{g} \mathrm{mL}^{-1}$ and incubated for $10 \mathrm{~min}$ in the dark at room temperature. After washing and resuspending again, the fluorescence intensity was measured in two technical replicates of $100 \mu \mathrm{L}$ (final resuspension) in black flat bottom 96 -well plates (at $30^{\circ} \mathrm{C}$, delay of $0.1 \mathrm{~s}$, gain 50 , excitation at $488 \mathrm{~nm}$ and emission at $525 \mathrm{~nm}$ ) using a Synergy MX microplate reader (BioTek, 258 Winooski, VT, U.S.A.). On the basis of the linear correlation between PHA fluorescence intensity and $\mathrm{mcl}$ PHA concentration final product concentrations could be quantified. Further details on $m c l$-PHA extraction procedure are given in the SI sec. S1.1.

Fluorescence microscopy images of $P$. putida KT2440 were acquired using an Axio Imager A2 fluorescence microscope (Carl Zeiss Microscopy GmbH, Jena, Germany). The staining of samples was carried out following the same procedure for mcl-PHA fluorescence measurements previously described. The cell suspensions 
were immobilized in $1 \%$ agarose, which was previously solidified and fixed on microscope slides.

Analytical Methods. Aromatic compounds were quantified by HPLC analysis in a Beckman System Gold 126 Solvent Module equipped with a System Gold 168 diode array detector (Beckman Coulter, Brea, CA, U.S.A.) and a reversed phase column (ISAspher 100-5 C18 BDS, ISERA, Düren, Germany). The method consisted of elution with $0.1 \% \mathrm{v} \mathrm{v}^{-1}$ trifluoroacetic acid (TFA) and $99.95 \% \mathrm{v} \mathrm{v}^{-1}$ methanol at a flow rate of $0.8 \mathrm{~mL} \mathrm{~min}^{-1}$ for $26 \mathrm{~min}$ at $40{ }^{\circ} \mathrm{C}$. Both solutions were applied at an initial ratio of 5:95 (methanol:TFA) for the first $2 \mathrm{~min}$. Subsequently, the proportion of methanol was linearly elevated to $25 \%$ during $18 \mathrm{~min}$ and then kept constant for $4 \mathrm{~min}$, before it was progressively reduced again to $5 \%$ in $2 \mathrm{~min}$. Aromatics were quantified with UV detection at a wavelength of $268 \mathrm{~nm}$ against standard curves prepared with commercial aromatic monomers.

Total ammonium nitrogen (TAN) was quantified using a Metrohm 761 compact ion chromatograph, equipped with a conductivity detector and a Metrosep C6-250/4.0 column (Metrohm, Switzerland). Alternatively, ammonium was also determined based on the Berthelot reaction. ${ }^{28}$

\section{RESULTS AND DISCUSSION}

Strain Screenings Reveal the Different Lignin Valorization Potentials of Wild Type Pseudomonas. Pseudomonas is well-known to include strains with a notable ability to mineralize aromatic compounds and transform them into valuable chemicals. ${ }^{29-33}$ While some studies incorporated several Pseudomonas putida strains into microbial screenings on lignin-derivatives, ${ }^{2,29,30,34-36}$ complementary experimental analysis to compare the actual valorization potential (i.e., biopolymer accumulation) of a broader selection of strains belonging to the genus Pseudomonas is still necessary. Thus, we implemented a 3-step sequential screening to select suitable Pseudomonas strains (Table S1) by evaluating their biodegradation and biotransformation capacity on lignin-model compounds. In this group, we included renowned strains in the field of lignin valorization like $P$. putida KT2440, ${ }^{5}$ strains with solvent tolerant characteristics (i.e., P. putida $S 12^{24}$ and $P$. taiwanensis VLB $120^{37}$ ), aromatic hydrocarbon catabolic features (i.e., P. putida $\mathrm{F}^{38}$ ) and strains that are well-known for their capacity to degrade polycyclic aromatics as well as their production of secondary metabolites (i.e., P. aeruginosa and $P$. fluorescens).

The first step evaluated the growth of the seven wild type Pseudomonas on 15 lignin model compounds at two concentrations ( 5 and $20 \mathrm{mM}$ ) under the same environmental conditions. The selection of the aromatic compounds was based on their prevalence in lignin depolymerization extracts or as intermediates found in aromatic upper pathways. ${ }^{39}$ Aromatics normally found after catalytic oxidation and depolymerization of lignin like vanillyl alcohol, ${ }^{40}$ acetovanillone, ${ }^{41}$ and 4-methylguaiacol ${ }^{42}$ were selected based on their challenging features for biodegradation. The results are shown as a heat map representation of the normalized bacterial growth after $39 \mathrm{~h}$ (Figure 1A). The normalization enabled quantitative and visual comparison of growth phenotypes. It gives values between 0 and 1 , in reference to the maximum scatter intensity values reached at 5 and $20 \mathrm{mM}$ substrate, which corresponds to $P$. putida KT2440 grown on benzoic acid and $P$. aeruginosa PAO1 grown on glucose, respectively (full data in Data Set S1).

About half of the tested lignin-derived monomers were well utilized for growth. The other half, poorly or not utilized, corresponds to phenols with aldehyde, ketone, and methoxy groups that are known to compromise the integrity of cell membranes. ${ }^{43}$ These groups have an increased inhibitory action compared to well-utilized phenolic acids. Recent efforts are trying to defeat this limitation by protein engineering of new promiscuous $\mathrm{P} 450$ aryl-O-demethylases and their heterologous expression in Pseudomonas putida. ${ }^{43,44}$

Unexpectedly, P. putida F1 was able to grow on sinapic acid, which to our knowledge has not yet been reported. Additionally, P. putida S12, which was one of the best performing strains overall, and $P$. taiwanensis showed changes in scattered light values for challenging molecules like syringol and 4-methylguiaiacol at low concentrations (5 $\mathrm{mM})$. However, a reliable cell growth was not possible for this strain on these molecules. Additional results and discussion for the new findings on P. putida F1 and S12 can be found in the SI sec. S3.1. P. taiwanensis VLB120 grew well with vanillic acid and 4-hydroxy benzoic acid at $20 \mathrm{mM}$, but it did not yield growth with $p$-coumaric and ferulic acid at this concentration, in contrast to the P. putida strains, which grew remarkably well. Intermediate performance values were obtained for $P$. putida KT2440. However, final growth values could have been underestimated due to morphological changes known to taking place after carbon depletion. ${ }^{29}$ Lastly, both strains of $P$. aeruginosa grew similarly and performed better than $P$. fluorescens, but less than the other strains.

Overall the best average normalized growth on lignin monomers at $20 \mathrm{mM}$ were achieved with $P$. putida S12, $P$. putida F1, P. putida KT2440, and P. taiwanensis VLB120 and thus, these four strains were selected for the next step to evaluate their valorization potential into mcl-PHAs.

This biopolymer is an intrinsic storage compound found in many Pseudomonas strains, which is produced under nutrient limitation. To induce mcl-PHA formation, two carbon to nitrogen ratios (nitrogen excess $\mathrm{C} / \mathrm{N}=4$ and nitrogen limited $\mathrm{C} / \mathrm{N}=40$ ) were tested in 48 -well flower plates providing suitable oxygen transfer conditions and a higher working volume for additional analysis. The aromatics p-coumaric, ferulic, benzoic and vanillic acid at $20 \mathrm{mM}$, each, were selected as single substrates to proceed.

Performance data to characterize the valorization potential are shown in Figure S4. Accumulation of mcl-PHA was promoted by reducing the initial amount of nitrogen source 10 -fold $(\mathrm{C} / \mathrm{N}=40)$. The optical density of the bacterial strains was affected under this nitrogen limited condition, although less for $P$. putida KT2440 (Figure S4A).

Interestingly, $P$. putida KT2440 also showed the highest valorization potential, described as the normalized mcl-PHA fluorescence in respect to the aromatic consumption (Figure S4B). The reduction of the nitrogen supply also had a positive effect on $P$. putida F1 mcl-PHA production, but almost no effect on P. putida S12. P. taiwanensis VLB120 showed low mclPHA production capacity on the aromatics, which it could utilize.

To conclude this part, our comparison of diverse Pseudomonas strains under identical conditions confirmed a high performance for aromatics degradation, especially for all three $P$. putida strains. Of them, the strain with the highest potential for lignin valorization under nitrogen limitations was P. putida KT2440. Therefore, only the three P. putida strains were included in step 3 to evaluate their aromatic funneling capacity from a defined aromatic mixture.

A mixture of $p$-coumarate, ferulate and benzoate further called CFB (each component at $10 \mathrm{mM}$ ) was selected as substrate with an even lower concentration of nitrogen, 


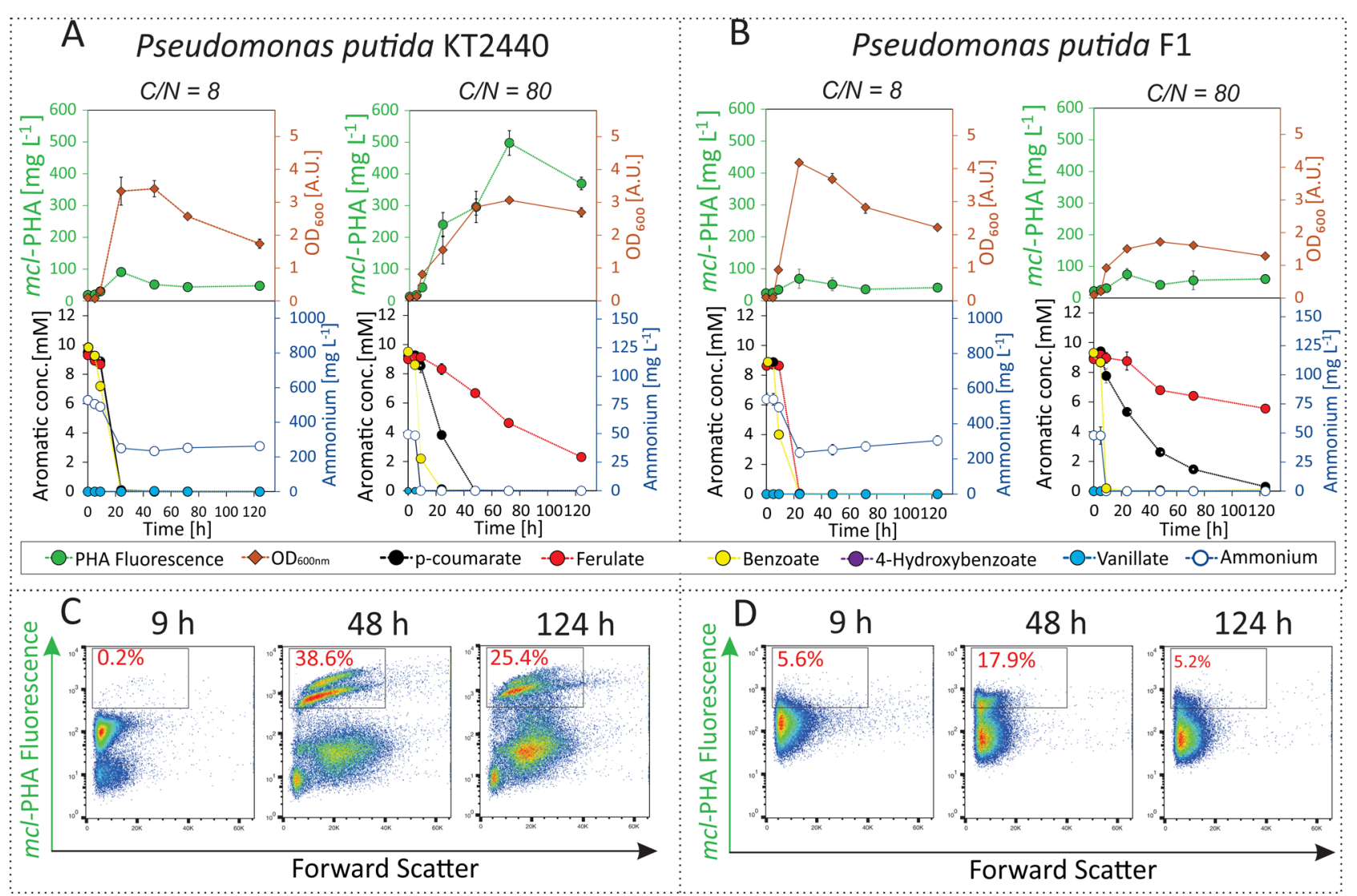

Figure 2. Conventional shake flask cultivations. Comparison of the aromatic funnelling capacity and $m c l-\mathrm{PHA}$ formation at two different nitrogen concentrations. The defined lignin-derived monomer mixture $(30 \mathrm{mM})$ consisted of $10 \mathrm{mM}$-coumarate, ferulate, and benzoate, each (dissolved in Hartman's mineral salt medium). Cell growth, $m c l$-PHA accumulation, aromatic consumption and ammonium utilization under nitrogen excess $(\mathrm{C} / \mathrm{N}=8)$ and nitrogen limitation $(\mathrm{C} / \mathrm{N}=80)$ for P. putida $\mathrm{KT} 2440(\mathrm{~A})$ and P. putida $\mathrm{F} 1$ (B). Flow cytometry analysis of the bacterial populations developed by both strains during nitrogen limitation $(\mathrm{C} / \mathrm{N}=80)$. Fluorescence scatter plots for selected time points, $P$. putida KT2440 (C) and P. putida F1 (D) during $124 \mathrm{~h}$. Error bars indicate the deviation of the mean $(n=2)$. The corresponding histograms of green fluorescence data for all the time points are shown in Figure S7.

corresponding to faster nutrient limitation $(\mathrm{C} / \mathrm{N}=80)$ (Figure $1 \mathrm{~B}$ and $\mathrm{C})$. As a control, the strains were also cultivated on the individual aromatics at the sum CFB aromatics concentration of $30 \mathrm{mM}$, each. The three components of the mixture were selected based on the growth performance and mcl-PHA accumulation, but also because they are representative ligninderived monomers of three important upper pathways during aromatic catabolism; the coniferyl and $p$-coumaryl branch of the protocatechuate node and the benzoate funnelling pathway of the catechol node.

Compared to the growth obtained at $20 \mathrm{mM}$ and $\mathrm{C} / \mathrm{N}=40$ (Figure S4A), the final $\mathrm{OD}_{600}$ values increased with more carbon source available, especially for $P$. putida KT2440 (approximately 2-fold) (Figure 1B). The normalized mcl-PHA fluorescence did not change with respect to the previous experiment with the single aromatic substrates (see Figure S4B vs Figure 1C). However, the intensity decreased in the cultivation with $P$. putida KT2440 for ferulate and $p$ coumarate, which could indicate a possible optimum condition around a $\mathrm{C} / \mathrm{N}$ of 40 for these single aromatics.

All three strains performed a diauxic shift around $5 \mathrm{~h}$, which can be related to the nitrogen depletion due to the stronger limitation $(\mathrm{C} / \mathrm{N}=80)$ (Figures $\mathrm{S} 5$ and $\mathrm{S} 6)$. For $P$. putida KT2440 grown in the CFB mixture, $\mu_{\max }$ lag phase, and final $\mathrm{OD}_{600}$ were overall not significantly altered compared to the values from growth on single aromatics (Figure 1B and Figure
S5A and B). In contrast, for the other two P. putida strains the $\mu_{(2) \max }$ values were affected for all aromatics (Figure S5D).

Quantitative biomass monitoring in microtiter plates is based on the linear correlation between scatter intensity and cell density, which has been validated even at high concentrations. $^{45,46}$ However, factors like cell morphological changes (size and shape) during cultivation and the effect of the mcl-PHA granules could hamper the practical comparison during screening campaigns. ${ }^{47}$ For this reason, aromatics consumption and mcl-PHA accumulation are more valid parameters to evaluate the success than biomass formation.

At this stage, our findings highlight that the funnelling capacity of the aromatics as well as the accumulation of $\mathrm{mcl}$ PHAs depends strongly on availability of essential nutrients. Therefore, we designed subsequent experiments in larger scale, where a hierarchical aromatic uptake under nutrient limited scenarios is possible to be discerned.

The Funneling Capacity in P. putida Is Affected When mcl-PHA Accumulation Is Triggered. To reveal the time course of the microbial activity under nitrogen limitation, conventional shake flask cultivations were carried out with the CFB mixture using $P$. putida KT2440 and P. putida F1 at two different nitrogen conditions (nitrogen excess $\mathrm{C} / \mathrm{N}=8$ and limitation $\mathrm{C} / \mathrm{N}=80$ ) (Figure 2). Under nitrogen excess, both strains consumed all aromatic compounds within $24 \mathrm{~h}$ utilizing $50 \%$ of the initial amount of ammonium. However, P. putida 


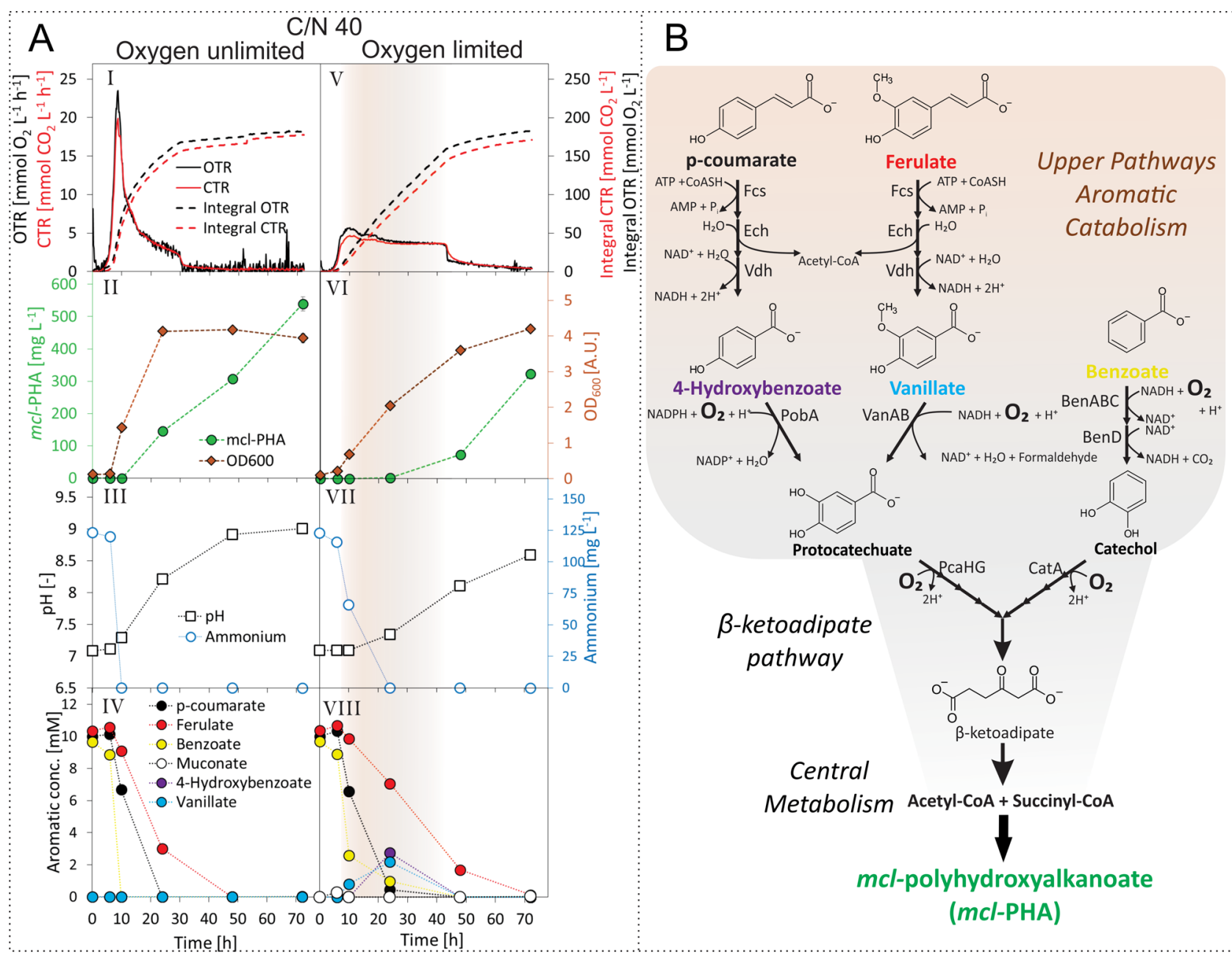

Figure 3. Comparison of the dynamics of aromatic funnelling into mcl-PHA in P. putida KT2440 under oxygen unlimited (I-IV) and limited conditions (V-VIII), both under nitrogen limitation at C/N 40 and CFB $30 \mathrm{mM}$ in Hartman's mineral salt medium (A). OTR $\mathrm{max}_{\max }$ of 23.5 and 5.7 mmol $\mathrm{O}_{2} \mathrm{~L}^{-1} \mathrm{~h}^{-1}$ were achieved for filling volumes of 10 and $50 \mathrm{~mL}$, respectively. Process parameters like the integral of OTR and CTR (I and V), $m c l-\mathrm{PHA}$ concentration and $\mathrm{OD}_{600}(\mathrm{II}$ and VI), $\mathrm{pH}$ and ammonium concentration (III and VII), and aromatic concentration (IV and VIII) are reported. The shaded area indicates intermediate accumulation and oxygen limitation. Representative pathways of biocatalytic funneling of the CFB aromatic mixture into $m c l$-PHA by P. putida KT2440 (B). ${ }^{29,31}$ Through catabolic upper-pathways the lignin monomers converge into a few central intermediates like catechol and protocatechuate, which are eventually cleaved and metabolized via the $\beta$-ketoadipate pathway to access the central carbon metabolism. Finally, part of the carbon flux excess accumulates as mcl-PHA under nitrogen limiting conditions. Fcs, feruloyl-CoA-synthase; Ech, feruloyl-CoA hydratase-lyase; Vdh, vanillin dehydrogenase; PobA, 4-hydroxybenzoate 3-monooxygenase; VanAB, vanillate O-demethylase; BenABC, benzoate 1,2-dioxygenase; BenD, benzoate 1,2-cis-dihydrodiol dehydrogenase; CatA, catechol 1,2-dioxygenase; and PcaHG, protocatechuate 3,4-dioxygenase.

F1 yielded higher $\mathrm{OD}_{600}$ values and degraded benzoate slightly faster than $P$. putida KT2440 during the first 9 h (50 vs $30 \%$ consumed, respectively) (Figure $2 \mathrm{~B}$ ). While a typical order of uptake preference is known for $P$. putida KT2440 when nitrogen does not limit growth: Benzoate $>p$-coumarate $>$ ferulate, no hierarchical aromatic uptake was observed (or resolved with the sampling frequency) with nitrogen excess. ${ }^{29}$ However, under nitrogen limitation, the aromatic uptake hierarchy clearly became evident and followed the classical regulatory control (i.e., catabolite repression by the $\mathrm{Crc}$ global regulator protein) known for $P$. putida. ${ }^{22,48,49}$ The consumption of benzoate was similar in both strains and correlated with the ammonium depletion. Afterward, P. putida KT2240 showed a faster consumption of the other two substrates. Under this condition, the $m c l$-PHA concentrations increased for $P$. putida KT2440 reaching a maximum of $497 \mathrm{mg} \mathrm{L}^{-1}$ after 72 h. Contrarily, P. putida F1 did not trigger mcl-PHA formation after entering nitrogen limitation and overall showed a drastic reduction in growth, which contrasted to the results of the screening step 3 .

The investigation of the dynamic mcl-PHA accumulation in both strains was complemented by flow cytometry (Figures 2C,D and S7). Initially, during the exponential growth of $P$. putida KT2440 (i.e., 9 to $24 \mathrm{~h}$ ), a narrow mcl-PHA fluorescence peak was observed (Figure S7). This indicates a similar $m c l$-PHA content within the mcl-PHA subpopulation. After $24 \mathrm{~h}$, when both benzoate and p-coumarate were consumed, the mcl-PHA-derived fluorescence peak became first wider and then split into two cell clusters, possibly indicating partial $m c l$-PHA degradation. A similar behavior has been previously reported by Karmann et al., ${ }^{50}$ who suggested a bistable behavior when $P$. putida KT2440 depolymerize $m c l$ PHA during carbon limitation. In this case, the limited consumption of ferulate and the lack of nitrogen could trigger 


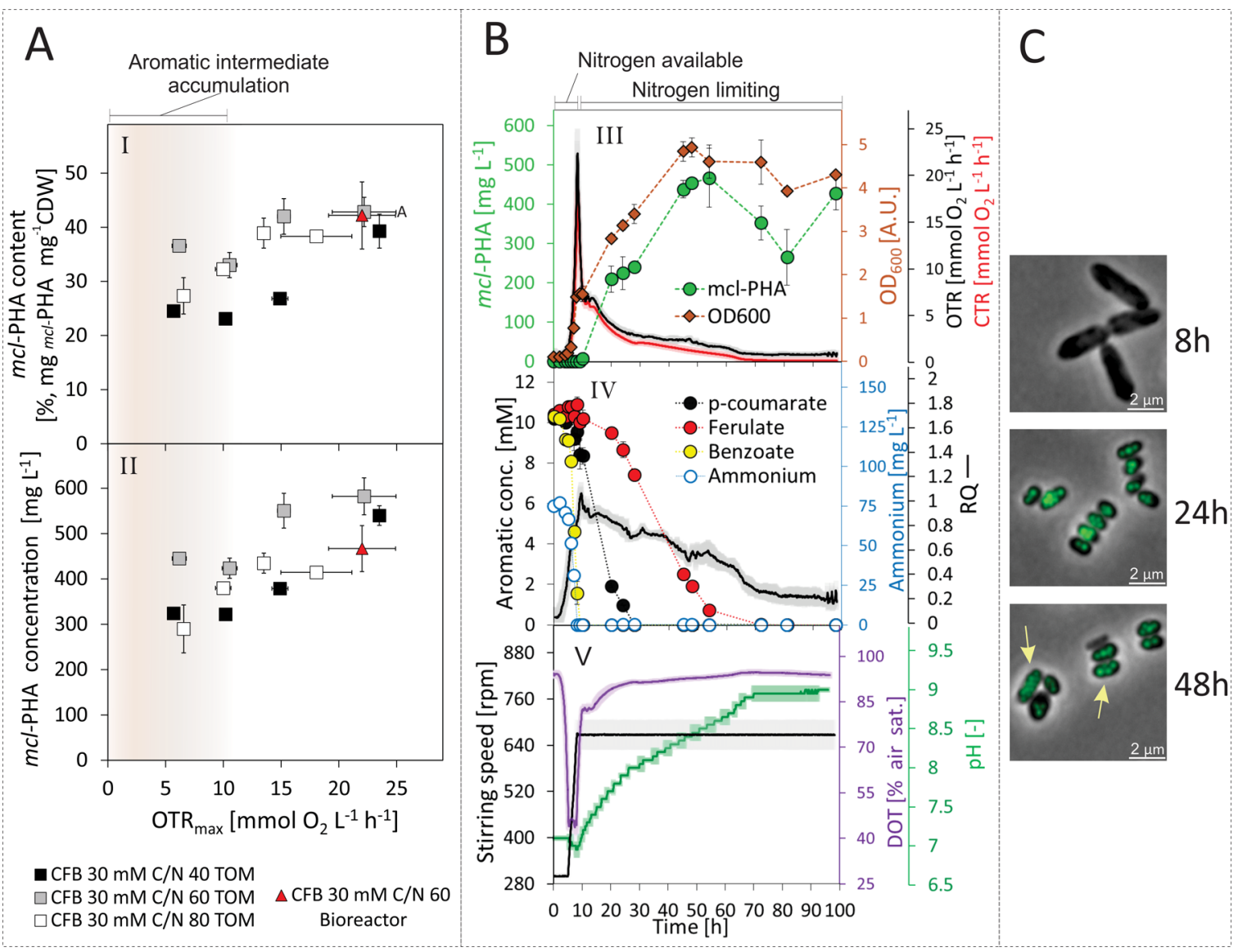

Figure 4. Overall correlation of online measurements of the respiratory activity $\left(\mathrm{OTR} \mathrm{max}_{\max }\right)$ and the $m c l$-PHA formation (i.e., content (I) and concentration (II)) in P. putida KT2440 carried out in TOM, an oxygen monitoring device for shake flasks (72h) and in a bioreactor (48h) (A). The shaded area indicates intermediates accumulation below values of $10 \mathrm{mmol} \mathrm{O} \mathrm{L}^{-1} \mathrm{~h}^{-1}$. Detailed bioprocess characterization of biocatalytic funneling of selected aromatic mixture CFB (prepared in Hartman's mineral salt medium) into mcl-PHA by P. putida KT2440 (OTR ${ }_{\max }$ of $22 \pm 2.9$ mmol $\mathrm{O}_{2} \mathrm{~L}^{-1} \mathrm{~h}^{-1}$ and $\mathrm{C} / \mathrm{N}$ of 60 ) in a bioreactor (B). The dynamic change of $m c l-\mathrm{PHA}$ concentration and $\mathrm{OD}_{600}(\mathrm{III})$, aromatic and ammonium concentration (IV) can be correlated to operational parameters like stirring speed, dissolved oxygen tension (DOT) and $\mathrm{pH}(\mathrm{V})$. The parameters OTR (III), CTR (III), and respiratory quotient (RQ) (IV) were calculated from off-gas composition analysis (SI sec. S2.5). Error bars indicate the deviation of the mean $(n=2)$. Microscopic captures of $m c l$-PHA accumulation at selected time points $(\mathrm{C})$. Arrows at $48 \mathrm{~h}$ indicate different number of $m c l$-PHA granules.

$m c l$-PHA depolymerization to provide cellular energy. On the contrary, mcl-PHA accumulating populations in P. putida F1 were only temporarily identified until $p$-coumarate was completely consumed, reaching a maximum of $17.9 \%$ of the total events at $48 \mathrm{~h}$ (see Figure 2D).

We hypothesized that oxygen availability could be responsible for the relevant differences found for $P$. putida F1 in shake flasks compared to the screening in 48-well flower plates. Therefore, we calculated the maximum oxygen transfer rate capacity $\left(\mathrm{OTR}_{\text {cap }}\right)$ for these systems (eqs S4 and S5 in SI sec S2.3 and S2.4). Theoretical maximum OTR $_{\text {cap }}$ of 40.9 and $16.1 \mathrm{mmol} \mathrm{L}^{-1} \mathrm{~h}^{-1}$ were found for the 48-well flower plates and shake flask, respectively, highlighting a great difference in maximum respiration capacity. The indifference of the $P$. putida KT2440 results also highlight the high tolerance of $P$. putida KT2240 toward stress generated during nitrogen depletion and lower OTRs, where mcl-PHA carbon storage formation occurs even although the funnelling capacity is affected. So besides a very good performance in funneling a mixture of aromatic compounds and the superior performance in mcl-PHA formation compared to all tested strains, P. putida KT2440 also presents the most robust biocatalyst against process parameter deviations. Our screening therefore clearly supports the focus of the research field on P. putida KT2440 for a direct native production of $\mathrm{mcl}$-PHA straight from mixed aromatics. Next, a detailed metabolic characterization of the funnelling process is performed for this strain.

Online Measurements of the Oxygen Consumption Reveal the Dynamics of Aromatic Funnelling into $\mathrm{mcl}-$ PHA in $P$. putida KT2440. To fine-tune the importance of the oxygen availability during nitrogen limitation on $m c l$-PHA accumulation, online measurements of the oxygen consumption at different combinations of oxygen and nitrogen availability were carried out to reveal the dynamics of aromatic funnelling into $m c l$-PHA during $72 \mathrm{~h}$ for P. putida KT2440. By changing the filling volumes $(10,20,30$, and $50 \mathrm{~mL})$ of shake flasks for each nitrogen condition ( $\mathrm{C} / \mathrm{N}$ ratios of 40,60 , and 80 ), the maximum oxygen transfer capacity (as $\mathrm{OTR}_{\text {cap }}$ ) was defined. The time-resolved dynamics of parameters evaluated for every $\mathrm{C} / \mathrm{N}$ ratio facilitated a detailed comparison. An example for one unlimited and one limited oxygen consumption under nitrogen limitation $(\mathrm{C} / \mathrm{N} 40)$ is shown in Figure 3A. The complete data set for all conditions can be found in Figures S8-S10.

The accumulation of $m c l$-PHA was affected negatively for all $\mathrm{C} / \mathrm{N}$ ratios while the maximum oxygen transfer capacity 
decreased (Figures S8-S10). The aromatic analysis of the offline samples permitted to identify an $\mathrm{OTR}_{\text {cap }}$ of approx-

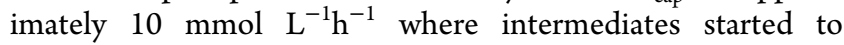
accumulate for every $\mathrm{C} / \mathrm{N}$ ratio (also see pathway Figure $3 \mathrm{~B}$ ). For values below this threshold, the simultaneous oxygen and nitrogen limitation became evident, where the shape of the OTRs curves presented a visible plateau, indicating a stable but low bacterial growth rate due to nutrient limitation. An example of this observation is shown in Figure $3 \mathrm{~A}$, where an OTR $_{\max }$ of 23.5 and $5.7 \mathrm{mmol} \mathrm{L}^{-1} \mathrm{~h}^{-1}$ were achieved at a C/N ratio of 40 for filling volumes of 10 and $50 \mathrm{~mL}$, respectively. Overall, parameters like $\mathrm{pH}, \mathrm{OD}_{600}$, and integrated OTR and CTR followed a similar trend, which is related to the intrinsic metabolic activity of aerobic growth. The consumption of ammonium correlated well with the benzoate uptake for all conditions according to the typical uptake priority of $P$. putida previously discussed.

Comparing the results with the previous flask cultivation $\left(\mathrm{OTR}_{\text {cap }}\right.$ of $16.1 \mathrm{mmol} \mathrm{L}^{-1} \mathrm{~h}^{-1}$ and $\mathrm{C} / \mathrm{N}$ ratio of 80 ), an underestimation of the real potential of $P$. putida KT2440 was exposed. The aromatic funneling profiles and the final $\mathrm{mcl}$ PHA concentration were well represented between the conditions 10 and $20 \mathrm{~mL}$ at a $\mathrm{C} / \mathrm{N}$ of $80\left(\mathrm{OTR}_{\max }=18 \pm\right.$ 3.1 and $13.5 \pm 0.4 \mathrm{mmol} \mathrm{L}^{-1} \mathrm{~h}^{-1}$, respectively) (Figure S10) but the maximum funneling performance of $P$. putida KT2440 was achieved at $\mathrm{C} / \mathrm{N}$ of 60 or 40 , where higher oxygen uptake rates were obtained. Hence, optimum conditions should be provided for a proper characterization or strain screening in nutrient limited lignin valorization studies. In this regard, reporting complete data of shaking conditions in conventional flask experiments is a priority to be able to compare results across studies.

An overarching correlation of the oxygen availability (based on $\mathrm{OTR}_{\max }$ ) and the most relevant bioprocess parameters (i.e., $m c l-P H A$ content and concentration) from this experiment is shown in Figure 4A (more details in Table S2). For both product parameters, the linear tendency indicates a positive effect of increasing the oxygen respiratory capacity on the $\mathrm{mcl}$ PHA accumulation for all evaluated nitrogen limited conditions. The biggest influence on mcl-PHA concentration was achieved for a $\mathrm{C} / \mathrm{N}$ ratio of 40 . Overall, the highest $\mathrm{mcl}$ PHA concentration $\left(582 \pm 41 \mathrm{mg} \mathrm{L}^{-1}\right)$ was obtained for a C/ $\mathrm{N}$ ratio of 60 at an $\mathrm{OTR}_{\max }$ of $22.2 \pm 2.8 \mathrm{mmol} \mathrm{L}^{-1} \mathrm{~h}^{-1}$. The catabolic funneling was affected for all conditions with a $\mathrm{C} / \mathrm{N}$ ratio of 80 , which resulted in residual amounts of ferulate that could not be converted into biomass and product (Figure S10 and Table S2). Thus, providing enough nitrogen for a correct function of the aromatic funneling pathways (i.e., enzyme synthesis) but sufficiently low to trigger $m c l$-PHA accumulation at high OTRs, can effectively reveal the real biocatalytic ability of promising strains with industrial relevance like $P$. putida KT2440. Therefore, increasing the oxygen supply in microbial cultivations is a proper strategy to overcome this trade-off that arises when the valorization of lignin needs to be performed under nitrogen limitation.

The delicate balance between carbon sources (i.e., ligninderived aromatic mixture), oxygen availability, and nitrogen supply is coordinated in P. putida by fine-tuning adjustments of several global regulatory networks, which are induced under specific environmental conditions. They control particular catabolic pathways to optimize carbon metabolism and energy generation. In order to avoid intermediate accumulation, the proper nutrient balance must be provided. For example, the hierarchy of aromatic uptake is known to be controlled by the Crc global regulator protein. ${ }^{22,48,49,51}$ Additionally, it has been also reported that this regulator inhibits $m c l$-PHA synthesis during exponential growth in media containing a balanced carbon source and nutrients. ${ }^{52}$ Cultivations with an equilibrated $\mathrm{C} / \mathrm{N}$ ratio and proper oxygen availability promote optimization of aerobic metabolism: Rapid initial growth and generation of an energy pool from benzoate reflects maximum energetic cost-benefit during nitrogen excess (i.e., feast condition). Later, the benefits are used to encounter nitrogen scarcity and more complex aromatic catabolism, with the result of boosted $m c l$-PHA accumulation.

But molecular oxygen is not only important as final electron acceptor of energy metabolism; it also plays an essential role for the corresponding oxygenases during the catalytic activation (i.e., debranching aromatic rings during the upper pathways) and cleavage of catechol and protocatechuate in the $\beta$-ketoadipate pathway (see oxygen-dependent reactions in Figure 3B). In addition, a simultaneous lack of nitrogen and oxygen could limit the activation or expression levels of enzymes in the $\beta$-ketoadipate pathway overall. Recently, outer membrane vesicles (OMVs) were reported to act as extracellular cargo of catabolizing enzymes that turnover model lignin-derived aromatic compounds in $P$. putida KT2440. ${ }^{53,54}$ Through proteomics characterization, these two studies found extracellular evidence of most of the enzymes that metabolize our CFB mixture. Thus, a deeper insight in this complex regulatory network toward microbial host optimization is still necessary.

Scale-Up Based on the Oxygen Transfer Rate. Finally, the optimum biocatalytic funneling of the CFB aromatic mixture into $m c l$-PHA by $P$. putida KT2440 was successfully scaled up and reproduced in a 1-L stirred tank bioreactor at an $\mathrm{OTR}_{\max }$ of $22 \pm 2.9 \mathrm{mmol} \mathrm{L}^{-1} \mathrm{~h}^{-1}$ and $\mathrm{C} / \mathrm{N}$ of 60 (Figure 4B). The higher working volume in the bioreactor permitted to verify the biomass, substrate and product dynamics observed in previous experiments without sampling limitation. The parameters OTR, CTR, and respiratory quotient (RQ) were calculated from off-gas composition analysis using eqs S6-S8 in SI sec. S2.5. The OTR $\mathrm{Tmax}_{\max }$ was obtained when ammonium was consumed, which correlated with a first stage of cell growth under nitrogen availability (i.e., the first $8 \mathrm{~h}$ ), a complete benzoate consumption, and a bigger cell size (see Figure 4C). At the same time, a diauxic shift, similar to the one observed in 48-well flower plates (see Figure S6), was associated with a change in the RQ. It reached a maximum value close to 1 and then decreased. This metabolic switch coincides with the sequential substrate utilization, changing from benzoate to $p$-coumarate. Additionally, it gives an idea of the metabolic reprograming from oxidative to reductive pathways (i.e., strong decrease in respiration).

After this point, $m c l$-PHA started to increase reaching a maximum concentration and content at $48 \mathrm{~h}$ (i.e., $467 \pm 51$

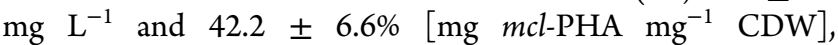
respectively) (Figure $4 \mathrm{~B} \mathrm{III}$ ). Compared to the shake flask experiment at the same $\mathrm{OTR}_{\max }$ and $\mathrm{C} / \mathrm{N}$ ratio, a faster dynamic of mcl-PHA evolution (i.e., faster increment and decrease of $m c l$-PHA after $72 \mathrm{~h}$ ) occurred in the fermenter. This could be related to different mixing patterns established in bioreactor vs shake flasks. This can influence a faster polymerization and depolymerization of $m c l-\mathrm{PHA} .{ }^{55,56}$ This is also supported by the different numbers of polymer granules observed in some bacterial cells (see arrows at $48 \mathrm{~h}$ Figure 
4C), which has been reported as bistable behavior of $P$. putida KT2440 depolymerizing $m c l$-PHA during carbon limitation. ${ }^{50}$

Depending on the type of substrate (and its initial concentration), mode of cultivation, and native or engineered strain, different $m c l$-PHA production performances have been reported in previous studies. For example, in the seminal work of Linger et al. ${ }^{5} 150 \mathrm{mg} \mathrm{L}^{-1} \mathrm{mcl}-\mathrm{PHA}$ at $36 \% \mathrm{CDW}$ was achieved from a representative aromatic substrate $\left(2 \mathrm{~g} \mathrm{~L}^{-1}\right)$ during a batch cultivation of a wild-type strain of $P$. putida KT2440. Recently, combining fed-batch cultivation and metabolic engineering of the same strain delivered $953 \mathrm{mg}$ $\mathrm{L}^{-1} \mathrm{mcl}$-PHA at $54 \% \mathrm{CDW}$ from $p$-coumarate (approximately $\left.4.2 \mathrm{~g} \mathrm{~L}^{-1}\right) .{ }^{11}$ The values obtained in this work $\left(582 \mathrm{mg} \mathrm{L}^{-1}\right.$ $m c l-\mathrm{PHA}$ at $43 \% \mathrm{CDW})$ from mixed lignin aromatics $\left(5 \mathrm{~g} \mathrm{~L}^{-1}\right)$ and batch cultivation of the native strain sit in the middle. However, through combining metabolic engineering and the presented process optimization strategy a further increase is very likely.

With a workflow involving different scale cultivations combined with online and offline performance analysis, we acquired valuable information about the biocatalytic capability in P. putida for funneling lignin-derived aromatics into $\mathrm{mcl}$ PHA. Through careful bioprocess observation and control, the performance of cells funnelling an aromatic mixture into $\mathrm{mcl}$ PHAs under nitrogen limitation became predictable. Thus, the complexity of dealing with heterogeneous lignin-derived aromatic mixtures and simultaneous nutrient limitations can be greatly reduced and reproducibility increased in future studies when warranting proper oxygen supply, especially during comparative microtiter and flask cultivations. In addition, by increasing the scale of production in bioreactors, the appropriate OTRs will be an important parameter for improving process performance and operational costs.

Despite the progress achieved in this study, there is still a long way toward achieving industrial relevant concentrations of lignin-derived PHA in a cost-effective and sustainable context. The PHA production at high levels has been established mainly from pure substrates like fatty acids, sugars, and alcohols or waste streams containing them. ${ }^{57}$ For instance, high cell densities between $100 \mathrm{~g} / \mathrm{L}$ and $115 \mathrm{~g} / \mathrm{L}$ (dry weight) containing approximately $46 \%$ polyhydroxybutyrate (PHB) on a dry-weight basis have been achieved from methanol, ${ }^{58}$ which is a nonfood substrate like lignin derivates. To compete with these types of substrates advances in biomass fractionation and lignin depolymerization are required promote the generation of less recalcitrant lignin-derived streams in future biorefineries.

Together with ongoing important metabolic engineering efforts for strain development on improving lignin aromatic uptake, balancing of carbon flux and avoiding mcl-PHA reassimilation, $^{8,11,19,20,51,55,56}$ we encourage future research on linking aspects like: media formulation (i.e., optimization of nutrients like nitrogen amount to ratio of real mixed lignin fractions), ${ }^{2}$ oxygen fine-tuned feeding strategies (i.e., DO stat fed batch cultivations) ${ }^{59}$ to improve $m c l$-PHA concentrations and minimize diauxic aromatic uptake profiles during different nitrogen availability (i.e., "feast and famine" stages), synergistic cocarbon feeding strategies to balance metabolic energy ${ }^{17,21,60}$ and ensuring final product quality (i.e., $m c l-\mathrm{PHA}$ material properties). ${ }^{16}$ The outcomes of this in depth bioprocess understanding may also benefit the production of other value compounds than mcl-PHA from lignin aromatics. For all future bioprocess engineering efforts, a balance between lignin, oxygen, and nitrogen supply will be essential. Eventually, this proposed combination of biocatalyst and bioprocess characterization will increase the efficiency and economics of lignin valorization in the next generation biorefineries.

\section{CONCLUSIONS}

This study describes the selection, comparison, and bioprocess characterization of proper microbial hosts of the genus Pseudomonas to accumulate mcl-PHA from a defined lignin derived mixture of p-coumarate, ferulate, and benzoate under nitrogen limitation. From strain-dependent differences observed in the lignin valorization potential, P. putida KT2440 emerged as a well performing and as the most robust biocatalyst. Through online measurements of the respiratory capacity of $P$. putida KT2440, we gained a comprehensive and detailed insight in how oxygen and nitrogen are the key factors to determine the success and efficiency of the bioprocess. While an induction of nitrogen limitation is critical for the timing and efficiency of $\mathrm{mcl}$-PHA production, sufficient availability of oxygen is essential for complete aromatics conversion and full metabolic activity. This knowledge permitted us to improve the mcl-PHA accumulation by up to $40 \%$. The final outcomes were scalable into 1-L stirred tank bioreactor based on the oxygen transfer rate, which represents a crucial step for further process optimization toward implementing sustainable bioconversions of lignin aromatics.

\section{ASSOCIATED CONTENT}

\section{SI Supporting Information}

The Supporting Information is available free of charge at https://pubs.acs.org/doi/10.1021/acssuschemeng.1c02682.

Materials and methods, calculations and results and discussion of the screening steps 1 and 2; additional figures showing a schematic overview of the experimental approach, bacterial growth model fit, definition of gates for flow cytometry, kinetic growth parameters during screening, histograms representing the dynamic change of mcl-PHA accumulating cell populations, and complete dynamics of aromatic funneling and $\mathrm{mcl}$-PHA accumulation at different $\mathrm{C} / \mathrm{N}$ ratios; tables describing details of the strains utilized and further bioprocess parameters; and data set $S 1$ includes all scatter values of the screening step 1 (PDF)

\section{AUTHOR INFORMATION}

\section{Corresponding Author}

Miriam A. Rosenbaum - Leibniz Institute for Natural Product Research and Infection Biology, Hans Knöll Institute (HKI), 07745 Jena, Germany; Faculty of Biological Sciences, Friedrich-Schiller-University Jena, 07743 Jena, Germany; ○ orcid.org/0000-0002-4566-8624;

Email: miriam.rosenbaum@leibniz-hki.de

\section{Authors}

Juan E. Ramírez-Morales - Institute of Applied Microbiology (iAMB), Aachen Biology and Biotechnology (ABBt), RWTH Aachen University, 52074 Aachen, Germany; Center for Microbial Ecology and Technology (CMET), FBE-Ghent University, 9000 Gent, Belgium; Leibniz Institute for Natural Product Research and Infection Biology, Hans Knöll Institute (HKI), 07745 Jena, Germany 
Phillip Czichowski - Institute of Applied Microbiology (iAMB), Aachen Biology and Biotechnology (ABBt), RWTH Aachen University, 52074 Aachen, Germany

Volkan Besirlioglu - Institute of Biotechnology, Aachen Biology and Biotechnology (ABBt), RWTH Aachen University, 52074 Aachen, Germany

Lars Regestein - Leibniz Institute for Natural Product Research and Infection Biology, Hans Knöll Institute (HKI), 07745 Jena, Germany; (1) orcid.org/0000-0003-1258-7171

Korneel Rabaey - Center for Microbial Ecology and Technology (CMET), FBE-Ghent University, 9000 Gent, Belgium

Lars M. Blank - Institute of Applied Microbiology (iAMB), Aachen Biology and Biotechnology (ABBt), RWTH Aachen University, 52074 Aachen, Germany; 10 orcid.org/00000003-0961-4976

Complete contact information is available at:

https://pubs.acs.org/10.1021/acssuschemeng.1c02682

\section{Notes}

The authors declare no competing financial interest.

\section{ACKNOWLEDGMENTS}

This work was supported by the European Union's Horizon 2020 research and innovation programme under the Marie Skłodowska-Curie grant agreement No 676070 (SuPER-W). This communication reflects only the author's view and the Research Executive Agency of the EU is not responsible for any use that may be made of the information it contains.

\section{REFERENCES}

(1) Zhang, C.; Wang, F. Catalytic lignin depolymerization to aromatic chemicals. Acc. Chem. Res. 2020, 53 (2), 470-484.

(2) Salvachúa, D.; Katahira, R.; Cleveland, N. S.; Khanna, P.; Resch, M. G.; Black, B. A.; Purvine, S. O.; Zink, E. M.; Prieto, A.; Martínez, M. J.; Martínez, A. T.; Simmons, B. A.; Gladden, J. M.; Beckham, G. T. Lignin depolymerization by fungal secretomes and a microbial sink. Green Chem. 2016, 18 (22), 6046-6062.

(3) Weiss, R.; Guebitz, G. M.; Pellis, A.; Nyanhongo, G. S. Harnessing the power of enzymes for tailoring and valorizing lignin. Trends Biotechnol. 2020, 38 (11), 1215-1231.

(4) Schutyser, W.; Renders, T.; Van den Bosch, S.; Koelewijn, S. F.; Beckham, G. T.; Sels, B. F. Chemicals from lignin: an interplay of lignocellulose fractionation, depolymerisation, and upgrading. Chem. Soc. Rev. 2018, 47 (3), 852-908.

(5) Linger, J. G.; Vardon, D. R.; Guarnieri, M. T.; Karp, E. M.; Hunsinger, G. B.; Franden, M. A.; Johnson, C. W.; Chupka, G.; Strathmann, T. J.; Pienkos, P. T.; Beckham, G. T. Lignin valorization through integrated biological funneling and chemical catalysis. Proc. Natl. Acad. Sci. U. S. A. 2014, 111 (33), 12013-8.

(6) Becker, J.; Wittmann, C. A field of dreams: Lignin valorization into chemicals, materials, fuels, and health-care products. Biotechnol. Adv. 2019, 37 (6), 107360.

(7) Salvachúa, D.; Johnson, C. W.; Singer, C. A.; Rohrer, H.; Peterson, D. J.; Black, B. A.; Knapp, A.; Beckham, G. T. Bioprocess development for muconic acid production from aromatic compounds and lignin. Green Chem. 2018, 20 (21), 5007-5019.

(8) Kohlstedt, M.; Starck, S.; Barton, N.; Stolzenberger, J.; Selzer, M.; Mehlmann, K.; Schneider, R.; Pleissner, D.; Rinkel, J.; Dickschat, J. S.; Venus, J.; van Duuren, J. B. J. H.; Wittmann, C. From lignin to nylon: Cascaded chemical and biochemical conversion using metabolically engineered Pseudomonas putida. Metab. Eng. 2018, 47, 279-293.

(9) Belda, E.; van Heck, R. G. A.; José Lopez-Sanchez, M.; Cruveiller, S.; Barbe, V.; Fraser, C.; Klenk, H.-P.; Petersen, J.; Morgat,
A.; Nikel, P. I.; Vallenet, D.; Rouy, Z.; Sekowska, A.; Martins dos Santos, V. A. P.; de Lorenzo, V.; Danchin, A.; Médigue, C. The revisited genome of Pseudomonas putida KT2440 enlightens its value as a robust metabolic chassis. Environ. Microbiol. 2016, 18 (10), 3403-3424.

(10) Poblete-Castro, I.; Binger, D.; Oehlert, R.; Rohde, M. Comparison of mcl-Poly(3-hydroxyalkanoates) synthesis by different Pseudomonas putida strains from crude glycerol: citrate accumulates at high titer under PHA-producing conditions. BMC Biotechnol. 2014, 14 (1), 962.

(11) Salvachua, D.; Rydzak, T.; Auwae, R.; De Capite, A.; Black, B. A.; Bouvier, J. T.; Cleveland, N. S.; Elmore, J. R.; Furches, A.; Huenemann, J. D.; Katahira, R.; Michener, W. E.; Peterson, D. J.; Rohrer, H.; Vardon, D. R.; Beckham, G. T.; Guss, A. M. Metabolic engineering of Pseudomonas putida for increased polyhydroxyalkanoate production from lignin. Microb. Biotechnol. 2020, 13 (1), 290298.

(12) Johnson, C. W.; Salvachúa, D.; Rorrer, N. A.; Black, B. A.; Vardon, D. R.; St John, P. C.; Cleveland, N. S.; Dominick, G.; Elmore, J. R.; Grundl, N.; Khanna, P.; Martinez, C. R.; Michener, W. E.; Peterson, D. J.; Ramirez, K. J.; Singh, P.; VanderWall, T. A.; Wilson, A. N.; Yi, X.; Biddy, M. J.; Bomble, Y. J.; Guss, A. M.; Beckham, G. T. Innovative chemicals and materials from bacterial aromatic catabolic pathways. Joule 2019, 3 (6), 1523-1537.

(13) Prieto, A.; Escapa, I. F.; Martínez, V.; Dinjaski, N.; Herencias, C.; de la Peña, F.; Tarazona, N.; Revelles, O. A holistic view of polyhydroxyalkanoate metabolism in Pseudomonas putida. Environ. Microbiol. 2016, 18 (2), 341-57.

(14) Wang, X.; Lin, L.; Dong, J.; Ling, J.; Wang, W.; Wang, H.; Zhang, Z.; Yu, X. Simultaneous improvements of cell growth and polyhydroxyalkanoate production from a lignin derivative for ligninconsolidated bioprocessing. Appl. Environ. Microbiol. 2018, 84 (18), No. e01469.

(15) Xu, Z.; Li, X.; Hao, N.; Pan, C.; de la Torre, L.; Ahamed, A.; Miller, J. H.; Ragauskas, A. J.; Yuan, J.; Yang, B. Kinetic understanding of nitrogen supply condition on biosynthesis of polyhydroxyalkanoate from benzoate by Pseudomonas putida KT2440. Bioresour. Technol. 2019, 273, 538-544.

(16) Mozejko-Ciesielska, J.; Szacherska, K.; Marciniak, P. Pseudomonas Species as Producers of Eco-friendly Polyhydroxyalkanoates. J. Polym. Environ. 2019, 27 (6), 1151-1166.

(17) Liu, Z.-H.; Shinde, S.; Xie, S.; Hao, N.; Lin, F.; Li, M.; Yoo, C. G.; Ragauskas, A. J.; Yuan, J. S. Cooperative valorization of lignin and residual sugar to polyhydroxyalkanoate (PHA) for enhanced yield and carbon utilization in biorefineries. Sustain. Energy Fuels 2019, 3 (8), 2024-2037.

(18) Jayakody, L. N.; Johnson, C. W.; Whitham, J. M.; Giannone, R. J.; Black, B. A.; Cleveland, N. S.; Klingeman, D. M.; Michener, W. E.; Olstad, J. L.; Vardon, D. R.; Brown, R. C.; Brown, S. D.; Hettich, R. L.; Guss, A. M.; Beckham, G. T. Thermochemical wastewater valorization via enhanced microbial toxicity tolerance. Energy Environ. Sci. 2018, 11 (6), 1625-1638.

(19) Borrero-De Acuña, J. M.; Gutierrez-Urrutia, I.; HidalgoDumont, C.; Aravena-Carrasco, C.; Orellana-Saez, M.; PalominosGonzalez, N.; van Duuren, J. B. J. H.; Wagner, V.; Gläser, L.; Becker, J.; Kohlstedt, M.; Zacconi, F. C.; Wittmann, C.; Poblete-Castro, I. Channelling carbon flux through the meta-cleavage route for improved poly(3-hydroxyalkanoate) production from benzoate and lignin-based aromatics in Pseudomonas putida H. Microb. Biotechnol. 2020 DOI: 10.1111/1751-7915.13705.

(20) Zhou, Y.; Lin, L.; Wang, H.; Zhang, Z.; Zhou, J.; Jiao, N. Development of a CRISPR/Cas9n-based tool for metabolic engineering of Pseudomonas putida for ferulic acid-to-polyhydroxyalkanoate bioconversion. Commun. Biol. 2020, 3 (1), 98.

(21) Xu, Z.; Pan, C.; Li, X.; Hao, N.; Zhang, T.; Gaffrey, M. J.; Pu, Y.; Cort, J. R.; Ragauskas, A. J.; Qian, W.-J.; Yang, B. Enhancement of polyhydroxyalkanoate production by co-feeding lignin derivatives with glycerol in Pseudomonas putida KT2440. Biotechnol. Biofuels 2021, 14 (1), 11 . 
(22) Rojo, F. Carbon catabolite repression in Pseudomonas: Optimizing metabolic versatility and interactions with the environment. FEMS Microbiol. Rev. 2010, 34 (5), 658-684.

(23) Blunt, W.; Sparling, R.; Gapes, D. J.; Levin, D. B.; Cicek, N. The role of dissolved oxygen content as a modulator of microbial polyhydroxyalkanoate synthesis. World J. Microbiol. Biotechnol. 2018, 34 (8), 106.

(24) Hartmans, S.; Smits, J. P.; van der Werf, M. J.; Volkering, F.; de Bont, J. A. M. Metabolism of styrene oxide and 2-phenylethanol in the styrene-degrading Xanthobacter strain 124X. Appl. Environ. Microbiol. 1989, 55 (11), 2850-2855.

(25) Karmann, S.; Follonier, S.; Bassas-Galia, M.; Panke, S.; Zinn, M. Robust at-line quantification of poly(3-hydroxyalkanoate) biosynthesis by flow cytometry using a BODIPY 493/503-SYTO 62 doublestaining. J. Microbiol. Methods 2016, 131, 166-171.

(26) Heyman, B.; Lamm, R.; Tulke, H.; Regestein, L.; Büchs, J. Shake flask methodology for assessing the influence of the maximum oxygen transfer capacity on 2,3-butanediol production. Microb. Cell Fact. 2019, 18 (1), 78.

(27) Kacmar, J.; Carlson, R.; Balogh, S. J.; Srienc, F. Staining and quantification of poly-3-hydroxybutyrate in Saccharomyces cerevisiae and Cupriavidus necator cell populations using automated flow cytometry. Cytometry, Part A 2006, 69A (1), 27-35.

(28) Krom, M. D. Spectrophotometric determination of ammonia: a study of a modified Berthelot reaction using salicylate and dichloroisocyanurate. Analyst 1980, 105 (1249), 305-316.

(29) Ravi, K.; Garcia-Hidalgo, J.; Gorwa-Grauslund, M. F.; Liden, G. Conversion of lignin model compounds by Pseudomonas putida KT2440 and isolates from compost. Appl. Microbiol. Biotechnol. 2017, 101 (12), 5059-5070.

(30) Ravi, K.; Garcia-Hidalgo, J.; Nobel, M.; Gorwa-Grauslund, M. F.; Liden, G. Biological conversion of aromatic monolignol compounds by a Pseudomonas isolate from sediments of the Baltic Sea. AMB Express 2018, 8 (1), 32.

(31) Nogales, J.; García, J. L.; Díaz, E. Degradation of aromatic compounds in Pseudomonas: A systems biology view. In Aerobic Utilization of Hydrocarbons, Oils and Lipids; Rojo, F., Ed.; Cham, 2017; pp 1-49.

(32) Kamimura, N.; Takahashi, K.; Mori, K.; Araki, T.; Fujita, M.; Higuchi, Y.; Masai, E. Bacterial catabolism of lignin-derived aromatics: New findings in a recent decade: Update on bacterial lignin catabolism. Environ. Microbiol. Rep. 2017, 9 (6), 679-705.

(33) Kumar, M.; You, S.; Beiyuan, J.; Luo, G.; Gupta, J.; Kumar, S.; Singh, L.; Zhang, S.; Tsang, D. C. W. Lignin valorization by bacterial genus Pseudomonas: State-of-the-art review and prospects. Bioresour. Technol. 2021, 320, 124412.

(34) Rodriguez, A.; Salvachúa, D.; Katahira, R.; Black, B. A.; Cleveland, N. S.; Reed, M.; Smith, H.; Baidoo, E. E. K.; Keasling, J. D.; Simmons, B. A.; Beckham, G. T.; Gladden, J. M. Base-Catalyzed Depolymerization of Solid Lignin-Rich Streams Enables Microbial Conversion. ACS Sustainable Chem. Eng. 2017, 5 (9), 8171-8180.

(35) Tomizawa, S.; Chuah, J.-A.; Matsumoto, K.; Doi, Y.; Numata, K. Understanding the Limitations in the Biosynthesis of Polyhydroxyalkanoate (PHA) from Lignin Derivatives. ACS Sustainable Chem. Eng. 2014, 2 (5), 1106-1113.

(36) Salvachúa, D.; Karp, E. M.; Nimlos, C. T.; Vardon, D. R.; Beckham, G. T. Towards lignin consolidated bioprocessing: simultaneous lignin depolymerization and product generation by bacteria. Green Chem. 2015, 17 (11), 4951-4967.

(37) Park, J.-B.; Bühler, B.; Panke, S.; Witholt, B.; Schmid, A. Carbon metabolism and product inhibition determine the epoxidation efficiency of solvent-tolerant Pseudomonas sp. strain VLB120 $\Delta$ C. Biotechnol. Bioeng. 2007, 98 (6), 1219-1229.

(38) Hughes, J. G.; Zhang, X.; Parales, J. V.; Ditty, J. L.; Parales, R. E. Pseudomonas putida F1 uses energy taxis to sense hydroxycinnamic acids. Microbiology (London, U. K.) 2017, 163 (10), 1490-1501.

(39) Xu, Z.; Lei, P.; Zhai, R.; Wen, Z.; Jin, M. Recent advances in lignin valorization with bacterial cultures: Microorganisms, metabolic pathways, and bio-products. Biotechnol. Biofuels 2019, 12, 32.
(40) Behling, R.; Valange, S.; Chatel, G. Heterogeneous catalytic oxidation for lignin valorization into valuable chemicals: what results? What limitations? What trends? Green Chem. 2016, 18 (7), 18391854.

(41) Das, L.; Xu, S.; Shi, J. Catalytic Oxidation and Depolymerization of Lignin in Aqueous Ionic Liquid. Front. Energy Res. 2017, 5 (21) DOI: 10.3389/fenrg.2017.00021.

(42) Kim, J.-Y.; Park, S. Y.; Choi, I.-G.; Choi, J. W. Evaluation of RuxNi1-x/SBA-15 catalysts for depolymerization features of lignin macromolecule into monomeric phenols. Chem. Eng. J. 2018, 336, $640-648$.

(43) Machovina, M. M.; Mallinson, S. J. B.; Knott, B. C.; Meyers, A. W.; Garcia-Borras, M.; Bu, L.; Gado, J. E.; Oliver, A.; Schmidt, G. P.; Hinchen, D. J.; Crowley, M. F.; Johnson, C. W.; Neidle, E. L.; Payne, C. M.; Houk, K. N.; Beckham, G. T.; McGeehan, J. E.; DuBois, J. L. Enabling microbial syringol conversion through structure-guided protein engineering. Proc. Natl. Acad. Sci. U. S. A. 2019, 116 (28), 13970-13976.

(44) Tumen-Velasquez, M.; Johnson, C. W.; Ahmed, A.; Dominick, G.; Fulk, E. M.; Khanna, P.; Lee, S. A.; Schmidt, A. L.; Linger, J. G.; Eiteman, M. A.; Beckham, G. T.; Neidle, E. L. Accelerating pathway evolution by increasing the gene dosage of chromosomal segments. Proc. Natl. Acad. Sci. U. S. A. 2018, 115 (27), 7105.

(45) Kensy, F.; Zang, E.; Faulhammer, C.; Tan, R.-K.; Büchs, J. Validation of a high-throughput fermentation system based on online monitoring of biomass and fluorescence in continuously shaken microtiter plates. Microb. Cell Fact. 2009, 8 (1), 31.

(46) Samorski, M.; Müller-Newen, G.; Büchs, J. Quasi-continuous combined scattered light and fluorescence measurements: A novel measurement technique for shaken microtiter plates. Biotechnol. Bioeng. 2005, 92 (1), 61-8.

(47) Kunze, M.; Roth, S.; Gartz, E.; Büchs, J. Pitfalls in optical online monitoring for high-throughput screening of microbial systems. Microb. Cell Fact. 2014, 13 (1), 53.

(48) Nichols, N. N.; Harwood, C. S. Repression of 4hydroxybenzoate transport and degradation by benzoate: a new layer of regulatory control in the Pseudomonas putida beta-ketoadipate pathway. J. Bacteriol. 1995, 177 (24), 7033-7040.

(49) Morales, G.; Linares, J. F.; Beloso, A.; Albar, J. P.; Martínez, J. L.; Rojo, F. The Pseudomonas putida Crc global regulator controls the expression of genes from several chromosomal catabolic pathways for aromatic compounds. J. Bacteriol. 2004, 186 (5), 1337-44.

(50) Karmann, S.; Panke, S.; Zinn, M. The bistable behaviour of Pseudomonas putida KT2440 during PHA depolymerization under carbon limitation. Bioengineering 2017, 4 (2), 58.

(51) Johnson, C. W.; Abraham, P. E.; Linger, J. G.; Khanna, P.; Hettich, R. L.; Beckham, G. T. Eliminating a global regulator of carbon catabolite repression enhances the conversion of aromatic lignin monomers to muconate in Pseudomonas putida KT2440. Metab. Eng. Commun. 2017, 5, 19-25.

(52) La Rosa, R.; de la Peña, F.; Prieto, M. A.; Rojo, F. The Crc protein inhibits the production of polyhydroxyalkanoates in Pseudomonas putida under balanced carbon/nitrogen growth conditions. Environ. Microbiol. 2014, 16 (1), 278-90.

(53) Salvachua, D.; Werner, A. Z.; Pardo, I.; Michalska, M.; Black, B. A.; Donohoe, B. S.; Haugen, S. J.; Katahira, R.; Notonier, S.; Ramirez, K. J.; Amore, A.; Purvine, S. O.; Zink, E. M.; Abraham, P. E.; Giannone, R. J.; Poudel, S.; Laible, P. D.; Hettich, R. L.; Beckham, G. T. Outer membrane vesicles catabolize lignin-derived aromatic compounds in Pseudomonas putida KT2440. Proc. Natl. Acad. Sci. U. S. A. 2020, 117, 9302.

(54) Choi, C.-W.; Park, E. C.; Yun, S. H.; Lee, S.-Y.; Lee, Y. G.; Hong, Y.; Park, K. R.; Kim, S.-H.; Kim, G.-H.; Kim, S. I. Proteomic characterization of the outer membrane vesicle of Pseudomonas putida KT2440. J. Proteome Res. 2014, 13 (10), 4298-4309.

(55) De Eugenio, L. I.; Escapa, I. F.; Morales, V.; Dinjaski, N.; Galán, B.; García, J. L.; Prieto, M. A. The turnover of medium-chainlength polyhydroxyalkanoates in Pseudomonas putida KT2442 and 
the fundamental role of PhaZ depolymerase for the metabolic balance. Environ. Microbiol. 2010, 12 (1), 207-221.

(56) Arias, S.; Bassas-Galia, M.; Molinari, G.; Timmis, K. N. Tight coupling of polymerization and depolymerization of polyhydroxyalkanoates ensures efficient management of carbon resources in Pseudomonas putida. Microb. Biotechnol. 2013, 6 (5), 551-563.

(57) Kourmentza, C.; Plácido, J.; Venetsaneas, N.; Burniol-Figols, A.; Varrone, C.; Gavala, H. N.; Reis, M. A. M. Recent Advances and Challenges towards Sustainable Polyhydroxyalkanoate (PHA) Production. Bioengineering 2017, 4 (2), 55.

(58) Bourque, D.; Pomerleau, Y.; Groleau, D. High-cell-density production of poly- $\beta$-hydroxybutyrate (PHB) from methanol by Methylobacterium extorquens: production of high-molecular-mass PHB. Appl. Microbiol. Biotechnol. 1995, 44 (3), 367-376.

(59) Poblete-Castro, I.; Rodriguez, A. L.; Lam, C. M.; Kessler, W. Improved production of medium-chain-length polyhydroxyalkanoates in glucose-based fed-batch cultivations of metabolically engineered Pseudomonas putida strains. J. Microbiol. Biotechnol. 2014, 24 (1), $59-69$.

(60) Liu, Z.-H.; Olson, M. L.; Shinde, S.; Wang, X.; Hao, N.; Yoo, C. G.; Bhagia, S.; Dunlap, J. R.; Pu, Y.; Kao, K. C.; Ragauskas, A. J.; Jin, M.; Yuan, J. S. Synergistic maximization of the carbohydrate output and lignin processability by combinatorial pretreatment. Green Chem. 2017, 19 (20), 4939-4955. 